\title{
La pourriture du cœur de l'Epicéa, Picea abies (L.) Karst. en Auvergne. Etude écologique
}

\author{
E. BENIZRY, G. DURRIEU, P. ROUANE \\ Laboratoire botanique et forestier, \\ Université P. Sabatier U.A CNRS 700, Toulouse
}

\begin{abstract}
Résumé
Ce travail, qui porte sur un échantillon de 63 placettes, étudie quelques-uns des facteurs susceptibles d'influencer le développement de la pourriture du çeur de l'Epicéa, essentiellement due à Heterobasidion annosum. Il montre qu'il est difficile d'apprécier l'importance de chacun d'eux pris isolément. Les analyses multifactorielles permettent de dégager un certain nombre de situations correspondant à des peuplements sains ou peu attaqués, ou au contraire dans lesquelles la pourriture est fréquente et grave.

L'importance particulière de l'âge des peuplements et surtout des coupes d'éclaircies est confirmée. De plus les facteurs édaphiques paraissent prépondérants, les sols sableux, dérivant de roches granitiques, acides, sont les plus favorables à un bon état sanitaire, tandis que les sols limoneux provenant de roches volcaniques, relativement peu acides prédisposent aux attaques. Un autre facteur important de sensibilisation est l'exposition en versant sud.
\end{abstract}

Mots clés : Pathologie forestière, pourriture du cœur, Picea, Heterobasidion.

\section{Introduction}

L'épicéa commun, Picea abies (L.) Karst. est une essence actuellement très répandue en France. Grâce à sa plasticité et sa croissance juvénile forte, il est largement utilisé dans les reboisements, aussi bien dans son aire naturelle (Alpes, Jura, Vosges) qu'en dehors, surtout dans les massifs montagneux, mais aussi à basse altitude.

Ainsi ses boisements développés dans des conditions très variées et dans certains cas très artificielles sont fragilisés et rendus plus vulnérables aux attaques parasitaires. En particulier, ils subissent parfois des dégâts intenses par pourriture rouge du cœur. Cette pourriture est attribuée le plus souvent aux attaques d'une Polyporacée, Heterobasidion annosum (Fr.) Bref. plus connu sous le nom de Fomes annosus (Fr.) Cooke (Delatour, 1972 ; Lanier et al., 1976). Le cycle du champignon est bien connu (RIsBeTh, 1959) de même que son mode de dispersion par germination des basidiospores sur les souches fraîchement coupées et transmission aux arbres sains voisins par les contacts racinaires. Ainsi le développement de la maladie est essentiellement lié aux coupes d'éclaircies et à l'âge des peuplements. Cependant d'autres facteurs nombreux et 
variés peuvent intervenir dans l'épidémiologie de la pourriture. Parmi ceux liés au peuplement ont été étudiés, la composition spécifique (Johanson, 1980 ; FlaCk, 1930 ; Werner, 1971), la densité (KANGas, 1952 ; KATo, 1967 ; HolmSGaARD et al., 1961), la nature de l'antécédent forestier ou agricole (REHFuess, 1973), l'influence des facteurs physiques a également été examinée par de nombreux auteurs : altitude (Populer, 1956 ; Moreau et Schaeffer, 1960 ; Maraitre, 1965), pente et exposition (...), nature, acidité et composition chimique du sol.

La grande variété de ces facteurs montre les difficultés qui se présentent à vouloir les étudier isolément. C'est l'une des raisons qui peut expliquer les contradictions qui apparaissent parfois entre les divers auteurs. Il vient s'y ajouter les origines géographiques très différentes des observations ou leur ponctualité. C'est ce qui a renforcé notre conviction de la nécessité d'une analyse multifactorielle de données recueillies sur un nombre assez élevé de stations.

\section{But de l'étude}

Le travail entrepris a pour but d'apprécier les relations pouvant exister entre les facteurs du milieu et l'importance de la pourriture rouge du cœur de l'Epicéa en Auvergne. En effet, sans qu'il existe une évaluation précise, les forestiers de la région considèrent qu'un nombre élevé de peuplements sont attaqués et fortement dépréciés du fait de l'intensité des dégâts.

Il est donc important, pour lavenir des jeunes peuplements et pour la conduite à tenir en cas de replantation, de savoir quels sont les facteurs qui favorisent l'installation et l'extension de la maladie ou au contraire permettent une meilleure résistance des arbres à l'invasion.

C'est pour ces raisons que le travail a porté sur une zone géographique relativement étendue, cela a permis d'obtenir un large éventail de situations différentes et d’observer ainsi des variations importantes des divers facteurs examinés.

Peut-on dire que notre travail consiste en fait à étudier l'écologie de l'Heterobasidion ? Cela reviendrait à postuler que toutes les pourritures rouges du cœur de l'Epicéa sont dues à ce champignon.

Les résultats obtenus par NkouAYA (1986) semblent infirmer cette hypothèse. Toutefois l'isolement d'un Basidiomycète autre que Heterobasidion annosum sur une souche coupée depuis un certain temps n'implique pas qu'il ait été à l'origine de la pourriture, il peut avoir supplanté $H$. annosum après l'abattage. On peut done considérer que la concordance Fomes-pourriture rouge est très élevée. Kallo et TammineN (1974) arrivent à la même conclusion. 


\section{Méthodes}

\subsection{Choix des placettes}

Nous avons effectué un échantillonnage volontaire stratifié. Les relevés sont implantés dans des zones que l'on estime représentatives et homogènes. Ainsi un nombre relativement réduit de relevés suffit pour couvrir l'échelle des variations de la zone d'étude.

Notre choix a été en outre déterminé par certaines considérations pratiques :

- l'étude portant sur une région relativement vaste où les peuplements d'épicéas sont dispersés, le repérage des placettes sur le terrain et leur accès sont difficiles ;

- pour déterminer sans risque d'erreur important la nature de la pourriture, il est nécessaire d'examiner des parcelles d'exploitation récente.

Ainsi nous avons observé 63 placettes dont la répartition est la suivante (fig. 1) :

- 2 relevés dans l'Allier,

- 11 relevés dans le Cantal,

- 27 relevés dans le Puy-de-Dôme,

- 23 relevés dans la Haute-Loire.

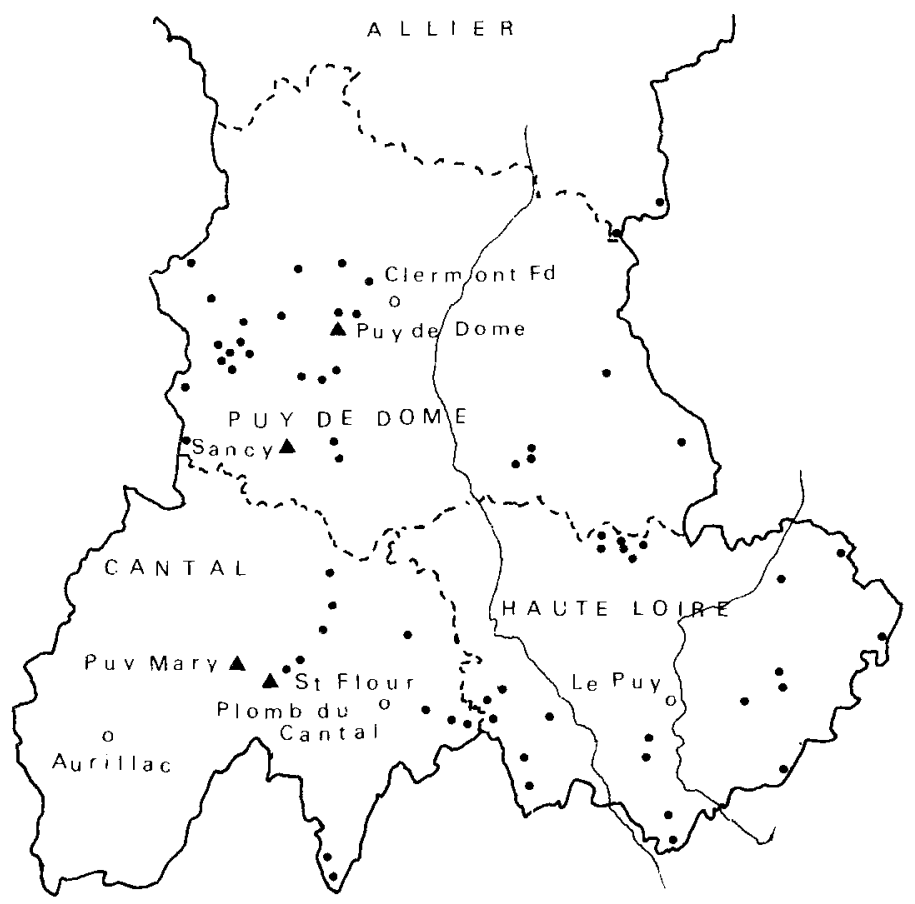

Fig. 1

Localisation des 63 placettes dans les 4 départements d'Auvergne.

Localization of the 63 spruce stands in the 4 departments of Auvergne. 


\subsection{Caractéristiques des placettes}

La délimitation des placettes a été réalisée de façon à obtenir la meilleure homogénéité possible quant aux facteurs les plus évidents: topographie, substrat, composition et densité du peuplement. Mais nous ne nous sommes pas fixés une surface donnée. En effet l'étude étant basée sur un comptage des souches, il était important de pouvoir en observer un nombre suffisant. Le nombre moyen observé par parcelle est de 31. Il est nettement inférieur à cette valeur pour quelques parcelles situées dans des peuplements entièrement sains, le total des souches observées s'élève à 1974.

\subsection{Données collectées}

Nous avons considéré tous les facteurs que l'on estime pouvoir être des variables explicatives. Ils sont de deux types : stationnels et forestiers.

\subsection{Données stationnelles}

\subsection{Topographie}

Il s'agit de : l'altitude (en mètres), la pente (en p. 100) et l'exposition.

\subsection{Roche mère et sol}

Parmi les critères édaphiques, on a distingué : la nature de la roche mère, la nature du sol, sa profondeur, son $\mathrm{pH}$, sa texture (pourcentages en argiles, limons et sables), et déterminé les pourcentages de certains éléments chimiques : carbone, azote, phosphore, potasse, magnésium, calcium.

\subsection{Données sylvicoles}

Sont notés : les données historiques (antécédent agricole ou forestier), l'âge des épicéas, la densité des peuplements (nombre de tige/hectare), la proportion nombre brins d'épicéas/nombre brins toutes essences (évaluées en dixièmes), la présence ou l'absence de coupes précédentes (éclaircies).

Les souches récemment coupées sont examinées et comptées afin d'estimer la fréquence des attaques et l'importance des altérations provoquées. Chaque souche a été classée en fonction de son degré d'altération, suivant une échelle de 0 à 6 .

- Stade 0 : bois sain, pas de décoloration anormale sur la section des souches.

- Stade 1: quelques taches brun-rouge seulement.

- Stade 2: nombreuses taches brun-rouge.

- Stade 3 : souches à bois creux dont le trou occupe moins du $1 / 4$ du diamètre soit $1 / 16$ de la section.

- Stade 4 : souches à bois creux dont le trou occupe entre le $1 / 4$ et la moitié du diamètre soit moins de $25 \%$ de la surface de la section.

- Stade 5 : souches à bois creux dont le trou occupe entre la moitié et les $3 / 4 \mathrm{du}$ diamètre, soit entre le $1 / 4$ et la moitié de la section.

- Stade 6: souches à bois creux dont le trou occupe plus des $3 / 4$ du diamètre, soit plus de la moitié de la section. 


\subsection{Exploitation des données}

\subsection{Variables à expliquer}

Le nombre de souches inventoriées n'étant pas constant d'une placette à l'autre, il a été nécessaire de raisonner en pourcentages et de déterminer pour chaque relevé le pourcentage de souches observées à chaque stade de dégradation. La présence de dégâts est évaluée de différentes façons :

- sur le simple critère d'absence-présence,

- en attribuant une note globale d'intensité des dégâts à chaque relevé. Cette note est calculée en additionnant les produits des pourcentages de souches de chaque stade par le numéro du stade.

Par exemple pour le relevé 22 nous avons :

56,52 p. 100 de souches saines (stade 0 )

34,78 p. 100 de souches au stade 1

8,7 p. 100 de souches au stade 3

soit une note globale de $34,78+(8,7 \times 3)=60,88(=61)$

\subsection{Variables explicatives}

La première idée est évidemment d'examiner l'influence, sur la pourriture, de chacun des facteurs pris séparément. Cette démarche ne peut donner des résultats entièrement valables que si tous les autres facteurs sont égaux. Cette condition est pratiquement irréalisable en travaillant sur des données de terrain, ou alors le nombre de parcelles répondant à cette exigence serait trop réduit pour avoir une valeur statistique.

C'est pour cela que nous avons privilégié l'analyse multifactorielle qui permet une perception globale de l'influence relative des différents facteurs. L'explication de certains des phénomènes observés peut alors être vérifiée en étudiant un facteur pris isolément.

\subsection{Méthodes d'analyses}

Deux méthodes d'analyse multivariée ont été appliquées. Ce sont l'analyse factorielle des correspondances (A.F.C.) qui a été appliquée aux variables qualitatives et l'analyse en composantes principales (A.C.P.) pour les variables quantitatives.

Afin d'obtenir une synthèse générale, nous avons regroupé les résultats significatifs des deux analyses précédentes, c'est-à-dire les 4 premiers axes de l'A.F.C. et les 3 premiers de l'A.C.P. les coordonnées des placettes sur ces divers axes ont été traitées par la méthode des groupements agglomératifs à lien complet (LEGENDRE, 1979). Cela a permis de réaliser un dendrogramme de l'ensemble des relevés. 


\section{Résultats et discussions}

\subsection{Analyses multifactorielles}

\subsection{Variables quantitatives}

Ces variables, au nombre de 10 , ont été traitées par une A.C.P. Le cercle de corrélation des variables dans le plan factoriel $1 / 2$ (fig. 2), qui exprime 46 p. 100 de l'information totale, met en évidence l'importance des facteurs pédologiques : les teneurs décroissantes en sable sont fortement liées à l'axe 1 tandis que celles en limon et en argile se trouvent à l'extérieur des 2 cadrans opposés. On voit également que les valeurs du $\mathrm{pH}$ sont liées négativement à l'axe 2 .

La position des relevés dans ce plan permet de distinguer deux zones très contrastées quant à l'intensité des attaques. L'une, centrée sur l'extérieur du cadran $(-1,+2)$, concentre l'essentiel des placettes indemnes $(10$ sur 16$)$ et la plupart des

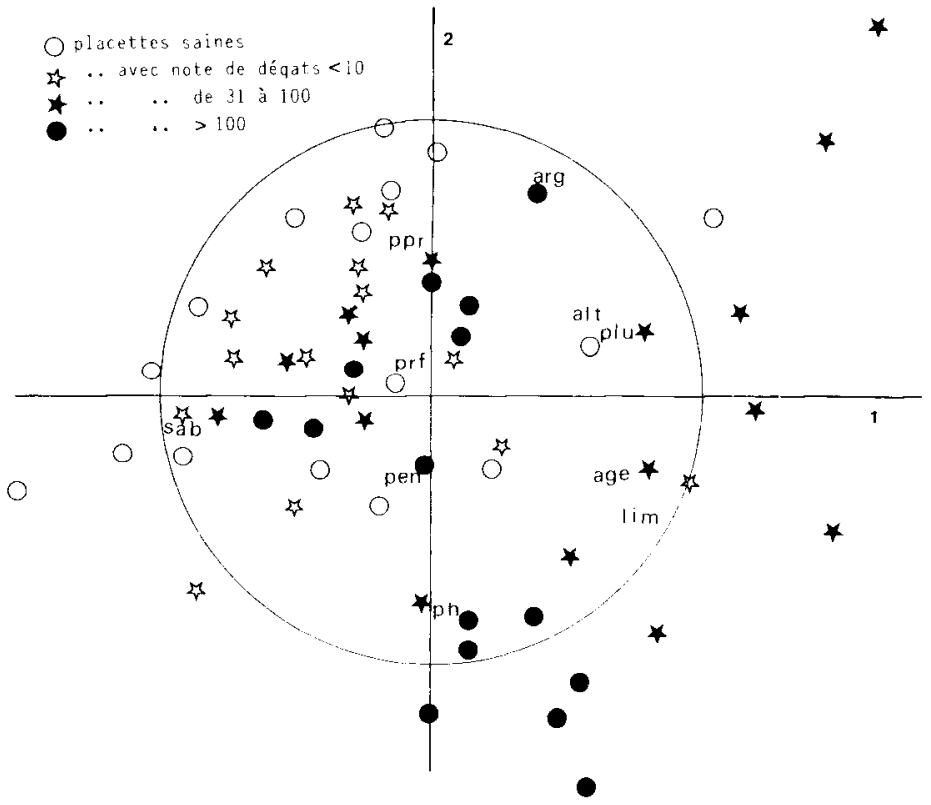

FIG. 2

Position des variables quantitatives, de leur cercle de corrélation et des placettes dans le plan factoriel 1-2 (A.C.P.).

Position of quantitative variables, of their correlation circle and of the plots in the factorial plane 1-2 (P.C.A.)

Légende des variables /

Caption of variables:

âge : âge / age ; alt. : altitude / altitude; arg : tencur en argile / clay amount ; lim : teneur en limon/silt amount; pen : pente / slope; $\mathrm{pH}$ : acidité / acidity :

$\mathrm{ppr}$ : proportion d'épicéa/spruce ratio ; prf : profondeur du sol/soil depth; sab : teneur en sable/ sand amount. 
autres ne sont que faiblement attaquées. L'autre, située à l'opposé ne contient pratiquement que des placettes attaquées. On peut même observer un noyau de 8 relevés concentré vers les valeurs négatives de l'axe 2 ( $\mathrm{pH}$ les moins acides) où les dégâts sont très élevés, note moyenne 176. Le premier secteur, à faible incidence du champignon correspond à des sols sableux ou argilo-sableux, très ou moyennement acides. Ces peuplements sont situés à basse altitude et sont jeunes. Le deuxième secteur rassemble des peuplements âgés croissant sur des sols limoneux, moyennement ou faiblement acides. Il est cependant intéressant de noter que la corrélation âge-maladie est toute relative. La distribution des placettes les plus fortement attaquées est assez quelconque par rapport à l'âge.

\subsection{Variables qualitatives}

Les variables non quantifiables ont été traitées à part dans une analyse factorielle des correspondances (fig. 3). Seuls les points ayant une bonne représentation dans le plan étudié ont été retenus. Le plan factoriel $(1,2)$ contient à lui seul une grande part de l'information: 64 p. 100. Il met en évidence l'opposition entre les roches mères (volcaniques ou cristallines) suivant l'axe 1 et les terrains plats ou en pente suivant l'axe 2.

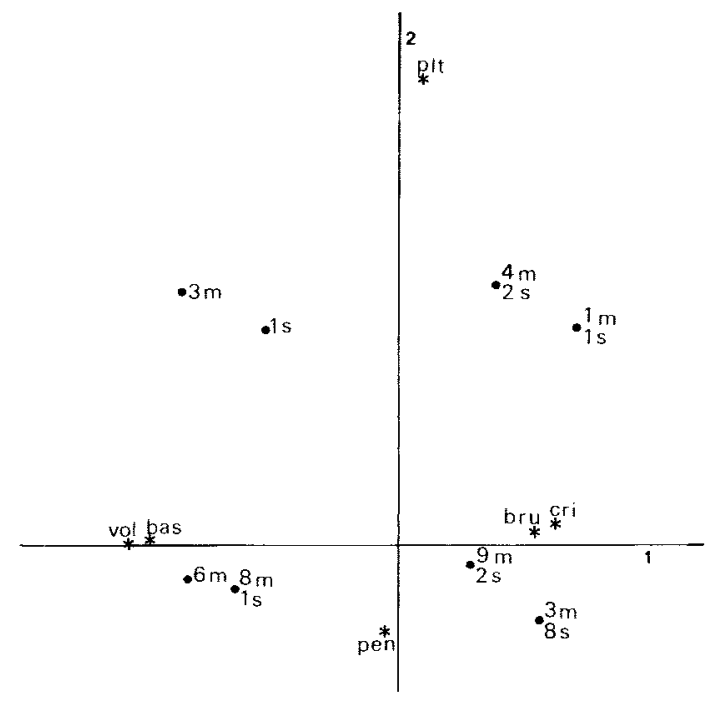

Fig. 3

Position des variables qualitatives et des placettes les mieux représentées dans le plan factoriel $1-2$ (A.F.C.). $2 S, 3 M$ : nombre de placettes saines et attaquées correspondant à un point.

Position of qualitative variables and plots with the best presence in factorial plane $1-2$ (F.C.A.).

$2 S, 3 M:$ number of healthy and attacked plots on one point of the plane.

Légende des variables /

Caption of variables:

bas : sols andiques / andic soils; bru : sols bruns / brown soils;

cri : roche mère cristalline / cristalline mother rock; pen : terrain en pente/sloping ground; plt : terrain plat / horizontal ground; vol : roche mère volcanique / volcanic mother rock. 
Ainsi la partie gauche du graphique rassemble essentiellement des relevés attaqués : 17 avec des notes d'intensité des dégâts élevées contre 2 indemnes, ils sont installés sur roche mère volcanique (et sols andiques). Par contre le plus grand nombre de parcelles saines se regroupe dans le cadran $(+1,-2)$ c'est-à-dire sols bruns dérivant de roche mère cristalline et en pente. Les parcelles atteintes qui se situent dans cette zone n'ont généralement subi que des dégâts plus réduits.

Les régions positives de l'axe 2 correspondent à des relevés sur terrain plat, avec une nette dominance de relevés où la pourriture est présente : 8 contre 4 indemnes.

Le plan factoriel $(3,4)$ (fig. 4) avec $34 \%$ de l'information fait ressortir l'influence des coupes d'éclaircies et de l'exposition. On constate bien l'importance des coupes dans l'installation de la maladie. La présence du champignon est très prédominante dans les relevés situés au voisinage du point $C_{+}: 20$ placettes contre seulement 4 indemnes. Par contre le quart du graphique où est situé le point $\mathrm{C}_{-}$regroupe 10 relevés sains à côté de 5 malades. L'axe explicatif des expositions regroupe toutes les placettes saines dans la zone correspondant aux expositions Nord, Est et Ouest, tandis que les expositions Sud (Sud-Est et Sud-Ouest) ne correspondent qu'à des relevés avec pourriture du cœur.

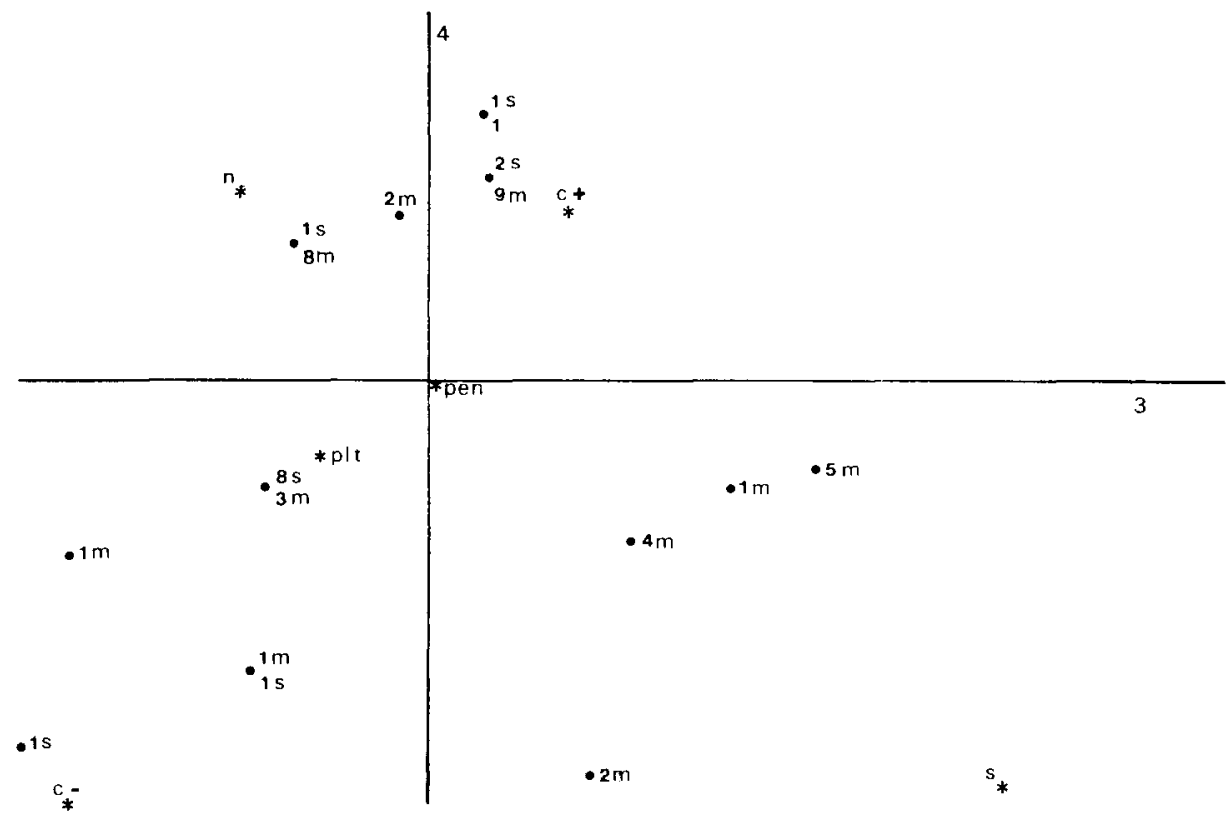

FIG. 4

Position des variables qualitatives et des placettes les mieux représentées dans le plan factoriel 3-4. Même légende que fig. 3, avec $C_{+}$: coupes d'éclaircies, $C_{-}:$pas de coupe d'éclaircie, $s$ : orientation sud $(S, S E, S W), n$ : autres orientations $(N, E, W, N E, N W)$.

Position of qualitative variable and plots with the best presence in factorial plane 3-4. Same caption as fig. 3 , with $C_{+}$: thinned stands, $C_{-}$: stands without thinning, $s$ : southerly exposure ( $S, S E, S W), n$ : other exposures $(N, E, W, N E, N W)$. 


\subsection{Analyse globale}

Il était évidemment intéressant de pouvoir réunir en une seule analyse synthétique les résultats précédents. La méthode des groupements agglomératifs à lien complet nous a permis de réaliser le dendrogramme de l'ensemble des relevés (fig. 5). En reportant les notes d'intensité des dégâts pour chaque station on peut ainsi délimiter des zones où les symptômes de pourriture sont faibles, moyennes ou élevées.

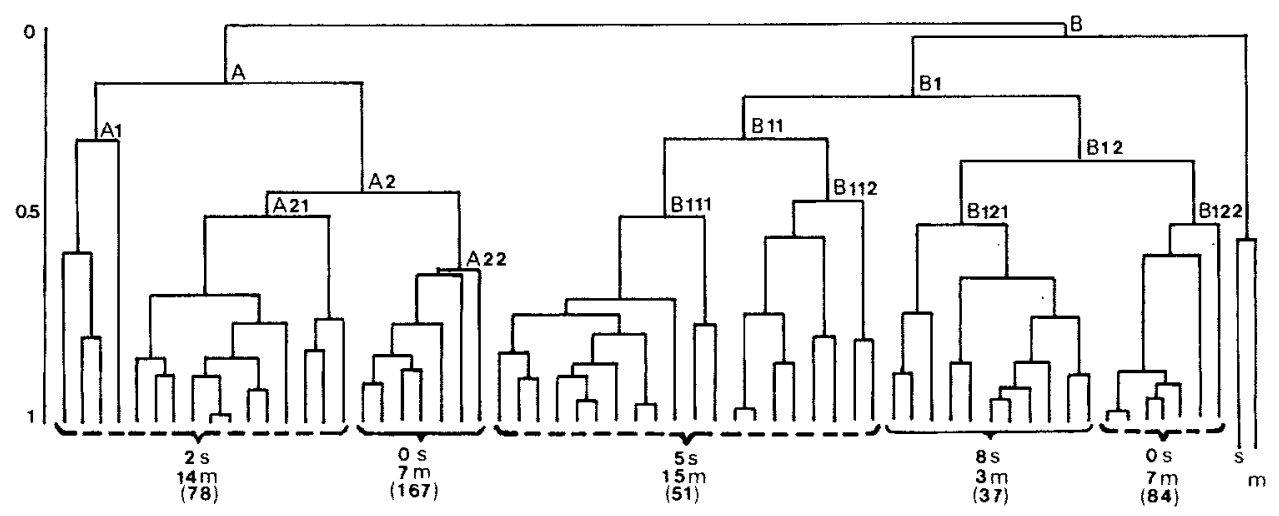

FIG. 5

Dendrogramme de l'ensemble des placettes. $2 S$ : nombre de placettes saines,

14M : nombre de placettes malades, (78) intensité moyenne des dégâts pour les placettes attaquées.

Dendrogram of the whole plots. $2 S$ : number of healthy plots, $14 M$ number of attacked plots,

(78) mean of decay strength on attacked plots.

La première disjonction sépare essentiellement les peuplements d'après la nature de la roche mère et par voie de conséquence des sols, le nœud $\mathrm{A}$ regroupe des relevés uniquement sur roche volcanique, $B$ ne contient que des relevés sauf un, sur roches cristallines ou cristallophylliennes avec les sols les plus acides. On constate que les différentes branches issues de $\mathrm{A}$ conduisent toutes sauf deux à des relevés ayant subi des attaques de Fomes. Elles sont relativement modérées à partir des nouds A 1 (parcelles n'ayant pas subi de coupes, installées à des altitudes relativement élevées sur sol argilo-limoneux) et A 21 (parcelles déjà soumises à des coupes, sur des terrains en pente non exposés au Sud sur sol sablo-limoneux). Sur les 16 parcelles concernées 2 sont saines et pour les autres la note moyenne d'intensité des dégâts est de 78 .

Par contre les dégâts sont élevés sur les 7 parcelles dérivant du nœud A 22 , avec une note moyenne de 167 , elles se distinguent surtout de celles issues de A 21 par leur exposition en versant Sud. A partir du nœud B, on note l'existence d'un lot important de relevés peu ou pas attaqués ( 8 parcelles sur 11 indemnes et note moyenne des dégâts des 3 hébergeant le Fomes $=37$ ). Ils correspondent au nœud B 121 c'est-à-dire à des peuplements jeunes n'ayant pas été éclaircis, situés sur des pentes non exposées au Sud.

De part et d'autre se trouvent deux groupes de stations nettement plus atteintes par la pourriture. Ce sont des peuplements déjà soumis à des coupes précédentes, les dégâts sont relativement limités sur les terrains plats ou non exposés au Sud (B 11 ) avec 5 indemnes et une note moyenne de 51 pour les 15 attaquées, plus importants en exposition Sud (B 122 ) avec une notation moyenne de 84. 


\subsection{Discussion}

A la lumière de ces différents résultats, il est intéressant d'analyser avec plus de précision l'influence des différents facteurs sur l'installation de la pourriture du cour, et de comparer nos observations avec celles des auteurs qui se sont intéressés à la question.

\subsection{Facteurs sylvicoles}

\subsection{Age et coupes}

Il nous a paru difficile de dissocier ces deux facteurs qui, pour les peuplements observés évoluent pratiquement dans le même sens. La figure 6 comme le plan factoriel 3-4 de l'A.F.C. (fig. 4) mettent en évidence l'influence des éclaircies. Sur ce graphique, nous avons considéré que tous les peuplements de plus de 60 ans avaient tous subi des éclaircies même si cela n’avait pas été noté.

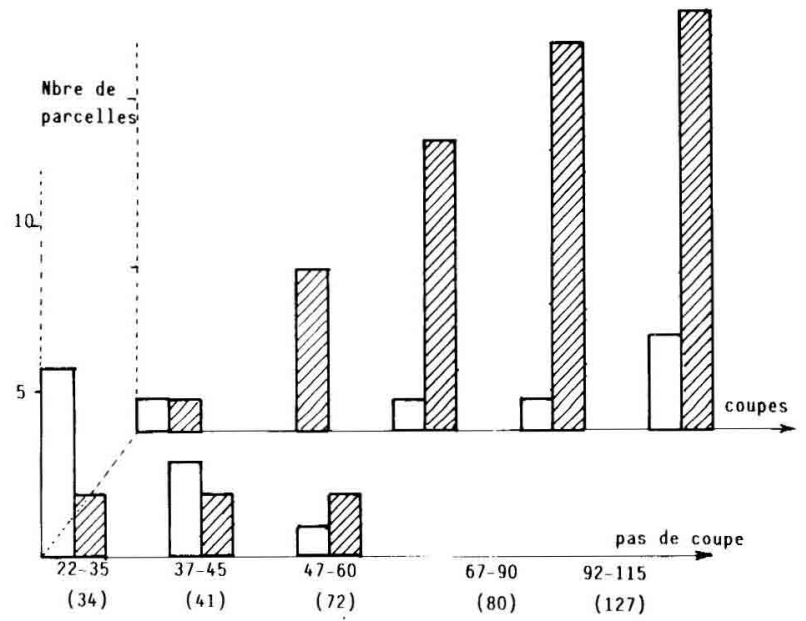

FIG 6

Influence des coupes d'éclaircies et de l'âge sur l'extension de la pourriture. (Entre parzwhìses, moyzme de l'intensité des dégâts pour les placettes attaguées).

Influence of age and thinnings on rot prevalence.

(Between brackets mean of decay strength on attacked plots).

Ainsi pour les peuplements de moins de 45 ans, on trouve 8 peuplements sains contre 4 attaqués en l'absence de coupe et 6 attaqués pour 1 sain après éclaircies. De même, on peut constater sur le dendrogramme (fig. 5) que le nœud B 121 , le plus sain, correspond uniquement à des parcelles n'ayant pas subi d'éclaircies. Tous les auteurs, depuis les observations de RisBeth (1951) s'accordent pour reconnaitre l'importance des coupes dans l'extension de la maladie (KARKKAINEN, 1969 ; C.E.T.E.G.R.E.F., 1978).

En revanche il n'est pas possible de mettre en évidence une influence de l'âge sur les peuplements de plus de 45 ans, les proportions d'arbres sains ou attaqués ne varient pas de façon significative. 
Cela ne signifie pas obligatoirement que les arbres les plus âgés deviennent plus résistants. Dans les parcelles anciennes seuls subsistent les mieux venus, qui ont été dominants pendant toute la durée du peuplement.

Est-ce leur vigueur qui leur a conféré une plus grande résistance, ou au contraire ont-ils été dominants parce que le Fomes ne les a pas attaqués? Il serait intéressant d'étudier la question en détail. Kallio \& Tamminen (1974) qui ont observé un phénomène analogue en Finlande, montrent que chez les arbres de 50 ans, l'accroissement en volume est beaucoup plus rapide pour les arbres sains que pour les arbres attaqués.

Cependant l'intensité des dégâts s'accroît assez régulièrement avec l'âge, en considérant les notes moyennes (fig. 6), mais avec des écarts considérables entre parcelles, par exemple 140 à 45 ans et 185 à 57 ans. La chose est bien mise en évidence par l'A.C.P. (fig. 2).

La proportion d'épicéas dans les peuplements mixtes, ne paraît pas corrélée avec la fréquence du parasite. Les observations antérieures (JoHANSSON, 1980 ; WeRNER, 1971) sont contradictoires. Dans notre cas, cela peut s'expliquer du fait que le plus souvent les essences compagnes sont des résineux, Pins ou Sapins, hôtes possibles pour le champignon.

\subsection{Facteurs topographiques}

\subsection{Altitude}

Le graphique d'A.C.P. montre une certaine relation entre l'altitude et la maladie.

Les stations saines ou peu attaquées se trouvent surtout à basse altitude. Pour les stations parasitées, l'intensité des dégâts paraît indépendante de ce facteur. La figure 7 illustre également ces constatations. Les attaques sont beaucoup plus fréquentes audessus de $1000 \mathrm{~m}$ environ, altitude pour laquelle l'intensité des dégâts est la plus marquée, tandis que plus haut, pour des fréquences très importantes les dégâts sont plus faibles.

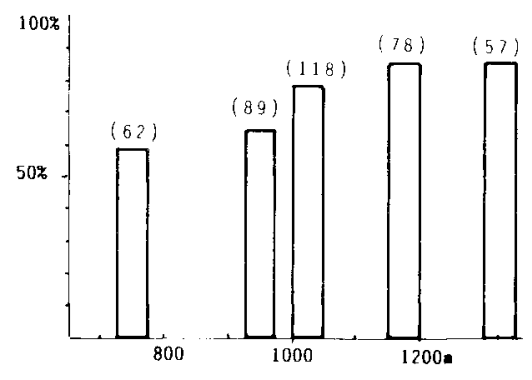

Fig 7

Proportion de placettes attaquées en fonction de l'altitude. (Entre parenthèses, moyenne de l'intensité des dégâts pour les parcelles attaquées).

Percentage of attacked plots according to their elevation. (Between brackets mean of decay strength on attacked plots). 
Ceci paraît en contradiction avec les données bibliographiques, mais qui correspondent à des régions ou tranches altitudinales différentes: Belgique (Maraitre, 1965 ; Populer, 1956) ou Jura (Moreau \& Schaeffer, 1960).

\subsection{Pente}

La position du facteur pente dans l'A.C.P. comme dans l'A.F.C. semble montrer que son influence est certainement faible. Effectivement la différence entre terrains plats, $67 \%$ d'infections et en pente, $77 \%$, est relativement peu importante. Elle s'accorde cependant avec les observations de divers auteurs (PoPuler, 1956 ; Powers, 1962 ; Huse, 1983). En revanche l'orientation de cette pente n'est pas indifférente. Les parcelles à exposition générale Sud (S., S.E. et SW) sont toutes attaquées, alors que pour les autres expositions, Nord, Est et Ouest, seulement $70 \%$ sont atteintes. Les dégâts les plus importants sont observés dans les parcelles exposées au Sud-Ouest. Dimitri (1969) avait fait la même constatation en Allemagne.

\subsection{Roche mère et sol}

Les précédentes analyses mettent en évidence une influence prépondérante des facteurs édaphologiques. Il y a une opposition très nette entre les peuplements sur roches cristallines et ceux sur roches d'origine volcanique. Les autres facteurs du sol, liés plus ou moins directement à ces origines (teneur en sable, $\mathrm{pH} . .$. ) jouent également un rôle considérable dans la structuration des analyses.

Il faut toutefois remarquer que d'autres facteurs peuvent venir surajouter leur effet. Ainsi dans l'A.C.P. l'axe 1 très influencé par la teneur en sable $(-0,91)$ et limon $(0,76)$ et donc par la nature de la roche mère est aussi assez représentatif des âges $(0,64)$. Il est donc nécessaire d'essayer de distinguer les effets de ces deux facteurs, leur corrélation venant essentiellement du fait que dans notre échantillonnage les peuplements les plus jeunes sont sur les terrains d'origine cristalline. Sur les roches basiques nous n'avons pas pu observer de parcelles de moins de 50 ans. Pour avoir une base de comparaison nous pouvons mettre en parallèle les valeurs obtenues au niveau du nœud A (terrains volcaniques) du dendrogramme avec celles du noud B (terrains cristallins) pour les peuplements de plus de 50 ans.

- En A, 2 sains et 21 malades avec une note moyenne de 107.

- En B, 4 sains et 15 malades avec une note moyenne de 85 .

On peut à l'intérieur de cet échantillonnage resserrer la comparaison sur des placettes présentant le maximum de similitudes. Ainsi quatre peuplements du nœud A 22, donc situés sur roche volcanique et en versant sud, dont les âges sont compris entre 57 et 100 ans (moyenne 85 ) présentent des dégâts dont la note moyenne est 151, tandis que les 4 parcelles du nœud B 122 , toujours exposées au Sud mais sur roche cristalline, avec des âges allant de 57 à 95 (moyenne 76) sont notés 99 pour les dégâts subits. Tout ceci confirme donc que les sols sur roche mère volcanique semblent favoriser l'extension du champignon et surtout le développement des dégâts. Nous n'avons pas trouvé d'étude comparable dans la bibliographie.

Risbeth (1951), Alexander et al. (1975) ont montré que les sols sablonneux à pH élevé et présentant un taux réduit de matière organique seraient favorables à la maladie. Ceci est en contradiction avec nos données sur le sable, mais concordant en ce qui concerne le $\mathrm{pH}$, en considérant que dans notre cas nous sommes toujours sur des terrains acides à $\mathrm{pH}$ inférieur à 6 . 
Les résultats des analyses chimiques des sols donnent des indications intéressantes en ce qui concerne les teneurs en phosphore et calcium. On note une élévation très nette de la proportion de souches pourries et surtout de l'intensité des dégâts dans les parcelles où les teneurs en ces deux éléments sont les plus élevées (fig. 8). Par contre nous n'avons pas constaté de corrélation avec les teneurs en azote et carbone.

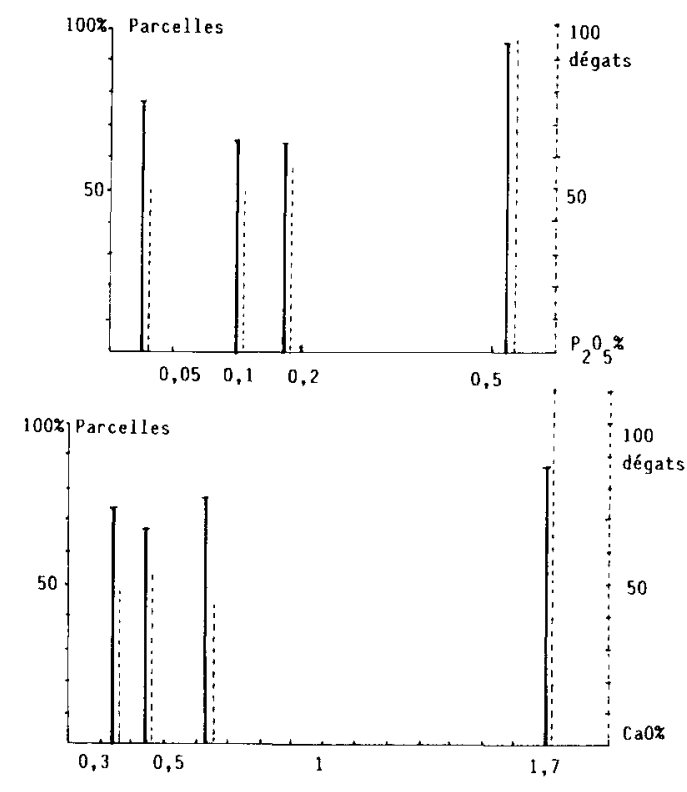

FIG. 8

Pourcentage des placettes attaquées et intensité des dégâts en fonction des teneurs en phosphore et calcium du sol.

Percentage of attacked plots and decay strength according to phosphorus and calcium amount in the soil.

Evers (1973) n'a pu établir de liaison entre l'intensité de l'infection et les rapports $\mathrm{C} / \mathrm{N}, \mathrm{C} / \mathrm{K}$ et $\mathrm{C} / \mathrm{P}$; mais il trouvait comme nous une corrélation positive avec la teneur en calcium. LAATSCH et al. (1968) et Rehfuess (1969) sont eux aussi d'accord pour ce dernier élément mais constatent une action favorable de l'azote sur le champignon.

Il paraît donc difficile, mis à part le calcium, de conclure de façon nette sur cette question.

\section{Conclusions}

Les différentes méthodes utilisées pour analyser l'ensemble des observations permettent, par une bonne concordance des résultats obtenus de montrer que pour la région considérée, il existe un certain nombre de facteurs qui favorisent l'installation de la pourriture à Heterobasidion annosum. Au contraire certaines conditions semblent 
permettre le développement et le maintien des peuplements dans un bon état sanitaire relatif. Les dégâts les plus notables sont observés sur les sols dérivant de roches volcaniques, à $\mathrm{pH}$ peu acide, riches en limon à des altitudes supérieures à 1000 mètres et en exposition Sud.

Par contre les attaques sont d'intensité plus faible sur terrains acides, riches en sable, provenant de roche mère cristalline, installés sur des pentes non exposées au Sud.

Dans tous les cas, comme on le savait déjà, l'influence des coupes d'éclaircies apparaît capitale dans l'extension de la maladie, cela devrait donc inciter les forestiers à faire appliquer de façon très stricte les traitements de protection sur les souches.

Si l'on peut donc tirer quelques conclusions intéressantes, cette étude montre aussi la complexité des interactions entre les divers facteurs. Elle peut ainsi servir de base de choix dans les critères d'échantillonnage pour de futurs travaux.

Reçu le 12 mars 1987.

Accepté le 22 décembre 1987.

\section{Remerciements}

Ce travail a été réalisé grâce à une subvention du Serfob d'Auvergne et de la Fédération départementale des groupements de défense des cultures du Puy-de-Dôme. Nous remercions également les ingénieurs et les agents de l'O.N.F. qui nous ont aidé et facilité le travail de terrain.

\section{Summary}

Heart rot of spruce, Picea abies (L.) Karst. in Auvergne. An ecological study.

This paper, which concerns a sampling of 63 plots, studies some of the factors capable of having an effect on the spruce heart rot development, mainly due to Heterobasidion annosum. It shows how much difficult it is to estimate the influence of each one of the factors. Multifactors analysis allow to take out some sites with healthy or light attacked stands or on contrary corresponding to stands suffering of frequent and heavy rotting.

Peculiar importance of age and thinnings is confirmed. Moreover edafic factors seem prevalent, sandy soils arising from granit like rocks, acid, are the most favourable, while silted soils from volcanic rocks, weakly acid are predisposing to attacks. Another main factor of susceptibility is southerly exposure.

Key words : Forest pathology, heart rot, Picea, Heterobasidion.

\section{Références bibliographiques}

Alexander S.A., Skfi.ty J.M. \& Morris C.I., 1975. Edafic factors associated with incidence and severity of disease caused by Fomes annosus in lobloly pine plantations in Virginia. Phytopathology, T. 65, 5, 585-591.

C.T.G.R.E.F. Grenoble, C.N.R.F. Nancy, 1978. La lutte préventive contre le Fomes annosus par le traitement des souches d'éclaircie. Rev. For. Fr., 30 (2), 124-129. 
Delatour C., 1972. Le Fomes annosus. Rev. For. Fr. $n^{\prime \prime} 1,20-38$.

Dimitri L., 1969. Untersuchungen über dic unteindischen Eintrittspforten der wichtigsten Rotfänkerreger bei der Fichte (Picea abies). Forstwiss. Cbl. 88, 281-308.

EVERS F.H., 1973. Zusammenhang zwischen chemischen Bodeneigenschaften und Kernfäulebefall in Fichtenbeständen. Mitt. Ver. Forstl. Standortskunde Forstpfl. Zücht, 22, 65-71.

Flack R., 1930. Neue Mitteilungen über die Rot-fäule. Mitt. Forstwirsch. Fortwiss, 1, 525-566.

Holmsgaard E., Holstenfr-Jorgensen H. \& Yde-Andersen A., 1961. Bodenbildung, Zuwachs und Gesundheitszustand vor Fichtenbeständen erster und zweiter Generation - 1 - NordSecland. Forstl. Forsogsv. Danm., 27, 1-167.

Huse K., 1983. Forekomst av rate i norsk granskog. (Frequency of butt rot in stands of Picea abies (L). Karst in Norway). Norsk Institutt for Skogsforskning. Rapport 83 (3), 39 p.

Johansson M., 1980. Svensk rotröteforskning i europeiskt sammanhang. Sveriges Skogsv. Förb. Tidskr. 4, 23-54.

Kallio T. \& Tamminen P., 1974. Decay of spruce [Picea abies (L.) Karst.] in the Aland Island. Acta For. Fenn., 138, $42 \mathrm{p}$.

Kangas E., 1952. Le Fomes annosus en Finlande : apparence, infection, dégâts. Inst. For. Fenn., $40(33), 34 \mathrm{p}$.

KÄrKKÄINEN M., 1969. Metsän vaurioituminen kesäaikaisessa puunkorjuussa. (The amount of injuries caused by timber transportation in the summer). Acta For. Fenn., 100, 35 p.

Kato F., 1967. Auftreten und Bedeutung des Wurzelschwammes [Fomes annosus ( $\mathrm{Fr}$ ) cooke] in Fichtenbestände Niedersachsens. Schr. Reihe Forstl. Fak. Univ. Göttingen, 39, 33-120.

LaAtsch W. \& Alcubilla W., 1968. Bezichungen zwischen dem standort und der kerfäule. Disposition der fichte. Forstwiss. Cbl., 87, 193-203.

Lanier L., Joly P., Bondoux P. \& Bellemère A., 1976. Mycologie et pathołogie forestière. T 2. Pathologie forestiere. Masson, Paris, $478 \mathrm{p}$.

Legendre L. \& Legendre P., 1979. Ecologie numérique. Masson, Paris.

Maraite H., 1965. Contribution à l'étude de la pourriture rouge de l'épicéa. Université Catholique de Louvain, 82 p.

Moreau R. \& SchaffFer R., 1960. Fomes annosus in the French Jura. Conf. and Study tour on fomes annosus (Inverness, Scotland).

Nkovaya B., 1986. Contribution à l'étude des champignons responsables de la pourriture du cceur des résineux en Auvergne. Rapport de D.E.A. Université de Clermont-Ferrand. INRA (non publié).

Populer CH., 1956. La pourriture rouge du cœur des résineux. Répartition de la maladie dans les pessières ardennaises ct essai d'interprétation écologique. Bul. Soc. Roy. Belge, 63 (7), 297329.

Powers H.R. \& Verral. A.F., 1962. A closer look at Fomes annosus. Forest Farmer, 3 p.

Reufuess K.E., 1973. Kernfäulbefall und Ernährungszurtand älterer Fichtenbestände in Wuchsgebiet «Baar-Wutach». Mitt. Ver. Forstl. Standortskunde Forstpfl. Zücht, 22, 9-26.

RISBETH J., 1951. Observations on the biology of Fomes annosus, with particular reference to East Anglian pine plantations. Ann. bot. N.S., 15, 1-21.

Risketh J., 1959. Dispersal of Fomes annosus and Peniophora gigantea. Trans. brit. mycol. soc., 42, 243-260.

WERNER H., 1971. Untersuchungen über die Einflüsse des Standorts und des Bestandesverhältnesse auf die Rotfäule (Kenfäule) in Fichtenbeständen der Mittleren Alb. Mitt. Ver. Forstl. Standortskunde Fortstpfl. Zücht, 20, 9-49. 